\title{
Using SXRR to Probe the Nature of Discontinuities in SLM Additive Manufactured Inconel 718 Specimens
}

\author{
RENÉ LAQUAI, BERND R. MÜLLER (D), JUDITH A. SCHNEIDER, \\ ANDREAS KUPSCH, and GIOVANNI BRUNO (B)
}

\begin{abstract}
The utilization of additive manufacturing (AM) to fabricate robust structural components relies on understanding the nature of internal anomalies or discontinuities, which can compromise the structural integrity. While some discontinuities in AM microstructures stem from similar mechanisms as observed in more traditional processes such as casting, others are unique to the AM process. Discontinuities in AM are challenging to detect, due to their submicron size and orientation dependency. Toward the goal of improving structural integrity, minimizing discontinuities in an AM build requires an understanding of the mechanisms of formation to mitigate their occurrence. This study utilizes various techniques to evaluate the shape, size, nature and distribution of discontinuities in AM Inconel 718, in a non-hot isostatic pressed (HIPed) as-built, non-HIPed and direct age, and HIPed with two step age samples. Non-destructive synchrotron radiation refraction and transmission radiography (SXRR) provides additional information beyond that obtained with destructive optical microscopy. SXRR was able to distinguish between voids, cracks and lack of melt in, due to its sensitivity to the orientation of the discontinuity.
\end{abstract}

https://doi.org/10.1007/s11661-020-05847-5

(C) The Author(s) 2020

\section{INTRODUCTION}

VARIOUS discontinuities can result from fabrication methods in which molten metal is solidified. During the AM process, voids can appear as they do in the case of cast components or fusion welded joints due to trapped gasses or gas formation during the process. Other discontinuities can arise from trapped particles or oxides. In AM processing, discontinuities such as porosity, voids, and inclusions occur but at a smaller, submicron scale than in traditional processes. ${ }^{[1-3]}$ In addition, other discontinuities unique to $\mathrm{AM}$ are found, including layer defects, lack of melt in, and entrapment of unconsolidated powders often resulting result from

RENÉ LAQUAI, BERND R. MÜLLER, and ANDREAS KUPSCH are with the Bundesanstalt für Materialforschung und prüfung (BAM), Unter den Eichen 87, 12205 Berlin, Germany. Contact e-mail: bernd.mueller@bam.de JUDITH A. SCHNEIDER is with the Department of Mechanical and Aerospace Engineering, University of Alabama in Huntsville, Huntsville, AL 35899. GIOVANNI BRUNO is with Bundesanstalt für Materialforschung und -prüfung (BAM) and also with the University of Potsdam, Institute of Physics and Astronomy, Karl-Liebknecht-Str. 24-25, 14476

Potsdam, Germany. Contact e-mail: Giovanni.bruno@bam.de

Manuscript submitted December 14, 2019.

Article published online May 30, 2020 non-optimized process parameters ${ }^{[3]}$ or irregularities in laser power.

Discontinuities can be characterized in a spatially resolved manner using classic two-dimensional (2D) image analysis techniques (e.g., based on optical or electron microscopy). These techniques can be expanded to three dimensions (3D) using destructive serial sectioning, often with focused ion beam (FIB) preparation. However, these destructive techniques are time-consuming and suffer from a limited field-of-view. To obtain (nearly) non-destructive 3D imaging, computed tomography $(\mathrm{CT})$ has been successfully used. ${ }^{[4-7]}$ Laboratory X-ray or Neutron CT can detect and image discontinuities down to 5 to $10 \mu \mathrm{m}$ in samples with dimensions about 5 to $20 \mathrm{~mm} \cdot{ }^{[4-6]}$ Recently, advanced parallel beam synchrotron computed X-ray tomography (SXCT) has been used. ${ }^{[8-10]}$ However, its spatial resolution, of approximately $1 \mu \mathrm{m}^{3}$ or slightly below, can only reliably identify volumetric discontinuities, and not other discontinuities such as cracks or inclusions. SXCT also requires miniature samples ${ }^{[10]}$ (cylinders with 1 to $2 \mathrm{~mm}$ diameter) to achieve the highest spatial resolution. In spite of the many advantages of SXCT, identifying micron size discontinuities in meso- or macro-sized samples becomes impracticable. 
A complementary route to detect microstructural features and discontinuities is represented by X-ray refraction techniques. In particular, using a synchrotron source, refraction radiography (SXRR) ${ }^{[11-13]}$ can be implemented. This technique has been successfully shown $^{[12]}$ to detect submicron inhomogeneities such as voids, cracks, and entrapped particles in bulk light materials such as ceramics and epoxy composites. ${ }^{[14]}$ Its main advantage over SXCT is the detectability of much finer discontinuities (even nano-sized) over much larger fields of view (centimeter size). Recently, the use of SXRR has been extended to metal matrix composites, ${ }^{[15]}$ metallic alloys ${ }^{[16]}$ and to determine the void shape and size in AM Titanium 6V/4Al. ${ }^{[17]}$ Previously, ${ }^{[10]}$ SXCT was compared with SXRR highlighting their complementary nature with regards to the detectability of submicrometer objects in macroscopic samples. It must be noted that a direct comparison is not possible, since the SXRR signal mostly comes from objects that are not detected in the CT scans.

In this study, the application of SXRR is used to characterize AM samples of Inconel 718, which has a higher density than other alloys investigated to date. In this study, SXRR was able to distinguish between discontinuities that resulted from lack of melt during the AM process, which were not sealed in subsequent HIP processing, from those of voids whose shape changed during HIP processing.

\section{FUNDAMENTALS OF X-RAY REFRACTION}

$\mathrm{X}$-ray refraction techniques were introduced a couple of decades ago, ${ }^{[12]}$ and have been successfully used for both materials characterization and non-destructive testing. ${ }^{[18]} \mathrm{X}$-ray refraction techniques such as X-ray refraction radiography (XRR) are used to obtain the amount of the relative internal specific surface, i.e., surface per unit volume, relative to a reference state of a sample. They are therefore a proven non-destructive characterization method to investigate and identify discontinuities such as inhomogeneities, inclusions, cracks and voids. Obviously, for practical reasons specimens are always reduced in size to fit the equipment, but the potential for fully non-destructive use of XRR has been repeatedly shown in the literature. ${ }^{[11,14,19]}$

X-ray refraction occurs whenever X-rays interact with interfaces between materials of different densities, as in the case of cracks, voids, or particles in a matrix. This is analogous to the behavior of visible light in transparent materials, such as lenses or prisms. Differences in the refraction indices between the two interfacing materials determine the refraction angle at the interface, which is also dependent on the wavelength of the radiation. Since the difference between the refraction indices of two neighboring materials for X-ray radiation is typically $10^{-5}$, X-ray optical effects can only be observed at very small scattering angles, which lie between several seconds and a few arc minutes. Since typical X-ray wavelengths are approximately $0.1 \mathrm{~nm}$, voids and cracks can be detected as soon as they exceed a size (or opening) of a few X-ray wavelengths in order for the wave to "notice" a density difference at the interface. That means that the smallest detectable object size is within the nanometer range. ${ }^{[9]}$ This is not to be confused with the spatial resolution or the size of objects that can be imaged. With the typical set-up at a synchrotron source, the spatial resolution is limited by the pixel size of the detector system. It must be also emphasized that because of the inevitable background noise, it is impossible to conclusively detect one single discontinuity. Therefore, a certain population of objects is necessary to yield an integrated signal above the background noise. XRR and SXRR yield a $2.5 \mathrm{D}$ signal that is integrated over the sample thickness. This results in the detection and imaging of populations rather than single discontinuities. While SXCT would yield truly 3D information, it would not be able to capture discontinuities below its spatial resolution; in this sense SXRR yields information (again, not images) on nano-sized objects but at a meso to macro length scale. This length scale matches that of many analytical models, so that the microstructural changes detected by SXRR have been quantitatively correlated with micromechanical models. ${ }^{[20]}$ Furthermore, X-ray techniques combining refraction and transmission are sensitive to the orientation of the discontinuity, thereby allowing the source of the discontinuity to be identified. ${ }^{[17,21]}$ For example, a spherical discontinuity would yield a refraction signal in any orientation of the sample. In contrast, cracks or elongated voids would yield a signal that strongly decreases when their surface normal is not parallel to the scattering vector of the instrumental set-up as identified by the bisectrix of the angle between the incident and refracted beam directions. Finally, it must be remarked that the X-ray refraction signal is sensitive to all kind of discontinuities (cracks, pores, grain boundaries) in a confounded manner. In practice, however, the largest contrast (difference in refraction index) is given by cavities and cracks, which are the most important discontinuities influencing the mechanical properties of materials.

\section{EXPERIMENTAL TECHNIQUES}

Micromelt Inconel 718 powders with a mesh size of $-325 \mathrm{M}$, or $44 \mu \mathrm{m}$, were used to fabricate SLM AM samples, using a Concept Laser M2 Cusing with a 1064 $\mathrm{nm}$ wavelength neodymium-doped yttrium aluminum garnet (Nd-YAG) laser. Based on prior optimization studies, which considered density and surface finish, the build parameters utilized in this study were: 180 Watt laser power, $600 \mathrm{~mm} / \mathrm{s}$ laser speed, $0.105 \mathrm{~mm}$ hatch spacing, and $0.035 \mathrm{~mm}$ layer thickness. Each layer was formed by the laser locally melting the powder using a pattern of alternately scanned $x$ and $y$ (i.e., in-plane perpendicular) directions. Cylindrical samples were printed in an $8 \times 8$ grid with a nominal $15 \mathrm{~mm}$ diameter, and $15 \mathrm{~mm}$ spacing, to a height of $200 \mathrm{~mm}$. Each 200-mm-long sample was cut into half to generate 128 cylinders, which underwent varying heat treatments prior to machining into sub-sized tensile samples. Each 
heat treatment lot was randomly chosen from the print grid to avoid biasing.

The size and shape analysis of the starting powders was carried out with a Horiba LA-9, laser diffraction particle size analyzer. The powders were measured dry with an air-jet dispersion method at $0.40 \mathrm{MPa}$ for 5000 seconds. Approximately 100 particles were used in the analysis. Additional imaging using a Keyence VK-X was correlated with the size and shape analysis obtained with the digital imaging process. The starting powder density was measured using He-Pycnometry.

Round sub-sized samples were used for tensile testing. A total of 6 samples were machined for each heat treatment outlined in Table I. Specimen 508 was given a full heat treatment common to post processing of Inconel 718. ${ }^{[2]}$ Due to the expected residual stresses, a stress relief and quench $(\mathrm{SR} / \mathrm{Q})$ is conducted prior to removal of the AM part from the build plate to minimize distortion. Hot isostatic pressing (HIP) is used to minimize volumetric voids. Due to the typical slow cooling in a HIP furnace, this is followed by a homogenization and quench (homo/Q) step to drive solidification phases back into solution with the matrix. A solutionizing and quench $(\mathrm{ST} / \mathrm{Q})$ is applied to precipitate some $\delta$ phase out along grain boundaries to minimize grain growth. After redistribution of the $\delta$ phase, an aging (Age) process is used to precipitate out the strengthening phases of $\gamma^{\prime}$ and $\gamma^{\prime \prime}$. In this study, a comparison was made between direct aging ${ }^{[23,24]}$ and the recommended two step aging per the SAE/ASM standard for wrought Inconel 718. ${ }^{\text {[22] }}$

The samples had a nominal $6.4 \mathrm{~mm}$ diameter and a 76 $\mathrm{mm}$ nominal length and the tensile axis aligned with the build direction $(Z)$. All tensile tests were conducted on a screw-driven mechanical actuator, which used a linear variable differential transformer for displacement feedback. Stress measurements were based on loads obtained from an $89 \mathrm{kN}$ load cell and sample dimension measurements. Strain measurements were obtained from a $25 \mathrm{~mm}$ extensometer calibrated to 50 pct strain. All tests were run in displacement control at a constant crosshead velocity of $1.3 \mathrm{~mm} / \mathrm{min}$.

After testing, sections were removed from the grip ends of the tensile samples oriented as shown in Figure 1(a). The optical microscopy (OM) samples were mounted in two orientations, parallel $(Z)$ and perpendicular to the build direction $(X-Y)$ and prepared using standard metallography practices. All samples were imaged using a Zeiss XioVert.A1m Inverted Microscope for Reflected Light Techniques. Bright field images were taken of un-etched samples to record the void size and morphology. Sufficient images were recorded at 200X magnification to obtain 100 voids rejecting indications of less than 6 pixels (or $2.7 \mu \mathrm{m}$ ) as noise. To reveal the grain structure, images were recorded for the samples etched with waterless Kalling's reagent. After etching the grain size was determined based on the Feret diameter using Image $\mathbf{J}$ analysis software.

Round cylinders nominally $2 \mathrm{~mm}$ diameter for XCT and flat slices nominally $9.5 \times 5.5 \mathrm{~mm}$ for SXRR studies were removed from samples \#508 and \#523 using wire EDM. To minimize the surface roughness, the flat samples were ground using $\mathrm{P} 4000 \mathrm{SiC}$ paper to a thickness of 0.160 to $0.180 \mathrm{~mm}$ for sample \#508 and 0.145 to $0.160 \mathrm{~mm}$ for sample \#523.

SXRR measurements were carried out at the BAM synchrotron laboratory BAMline at Helmholtz-Zentrum Berlin, Germany. ${ }^{[25,26]}$ The two prepared samples were mounted in a slide frame as shown in Figure 2.

A highly collimated monochromatic X-ray beam with an energy bandwidth of 0.2 pct was used. ${ }^{[2,27]}$ The beam energy was set to $30 \mathrm{keV}$ to achieve a sample X-ray transmission of about 25 pct (on the $0.2 \mathrm{~mm}$ thick plates). A pco.1600-Camera $(1600 \times 1200$ pixel $)$ in combination with a lens system and a $50 \mu \mathrm{m}$ thick CWO scintillator screen provided a pixel size of $4 \mu \mathrm{m} \times 4 \mu \mathrm{m}$. The incident beam was narrowed to the field-of-view of about $6.3 \mathrm{~mm} \times 4.7 \mathrm{~mm}^{[27]}$ by a slit system to avoid detector backlighting. ${ }^{[28]}$

In contrast to transmission-based radiographic measurements, a Si (111) analyzer crystal was placed in the beam path between the sample and the camera system to perform refraction radiographs as shown in Figure 2. The analyzer crystal reflects the beam transmitted by the sample into the detector system, if the incidence angle is set to the Bragg angle $\left(\theta_{\mathrm{B}}=3.779 \mathrm{deg}\right.$ at $\left.30 \mathrm{keV}\right)$. By tilting the analyzer crystal around an axis perpendicular to the scattering plane, a rocking curve is recorded. This describes the scattered beam intensity as a function of the deviation from the Bragg angle, $\Delta \theta=\theta-\theta_{\mathrm{B}}$. The rocking curve was recorded for each sample by taking 41 radiographs between $\theta=3.775 \mathrm{deg}$ and $\theta=3.783 \mathrm{deg}$ with a step size of $\Delta \theta=0.0002 \mathrm{deg}$ and exposure time of 5 seconds. Both samples were measured in two orthogonal orientations, with their build direction $(Z)$ parallel and perpendicular to the scattering vector. In addition, the following images were acquired: dark field (beam off) and flat field (beam on, but without sample). The dark field image is used to subtract the dark current and detector readout noise from the sample and flat field acquisitions. The flat field images quantify the instrumental artifacts and noise and are used to correct the $\mathrm{X}$-ray refraction radiography images of the sample.

Table I. Heat Treatment Parameters (Temperature ${ }^{\circ} \mathrm{C} /$ time h) ${ }^{[23,24]}$

\begin{tabular}{lllllll}
\hline Sample ID & SR/Q & HIP/SC & Homo/Q & ST/Q & Age 1 \\
\hline 554 & - & - & - & - & - & $-160 / 10$ \\
508 & $1066 / 1$ & $1163 / 3$ & $1163 / 1$ & $1066 / 1$ & $649 / 10$ \\
523 & - & - & - & $1010 / 1$ & $718 / 2$ & - \\
\hline
\end{tabular}



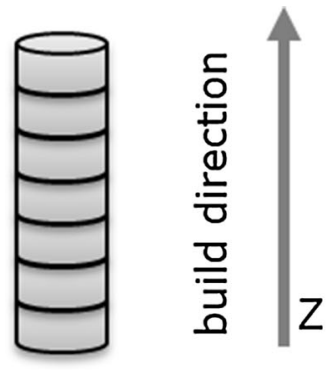

Z

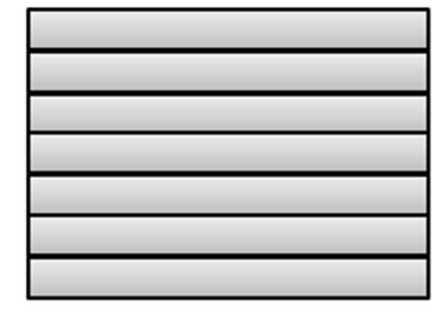

(a)

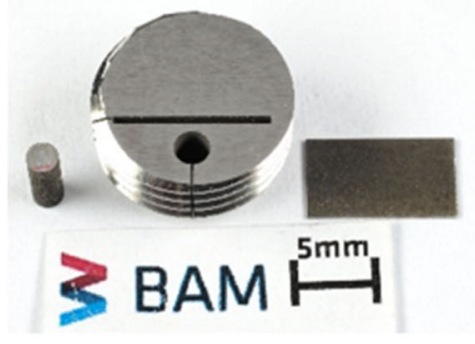

(b)

Fig. 1-Build direction of samples in this study (a); location of SXRR and OM samples (b).

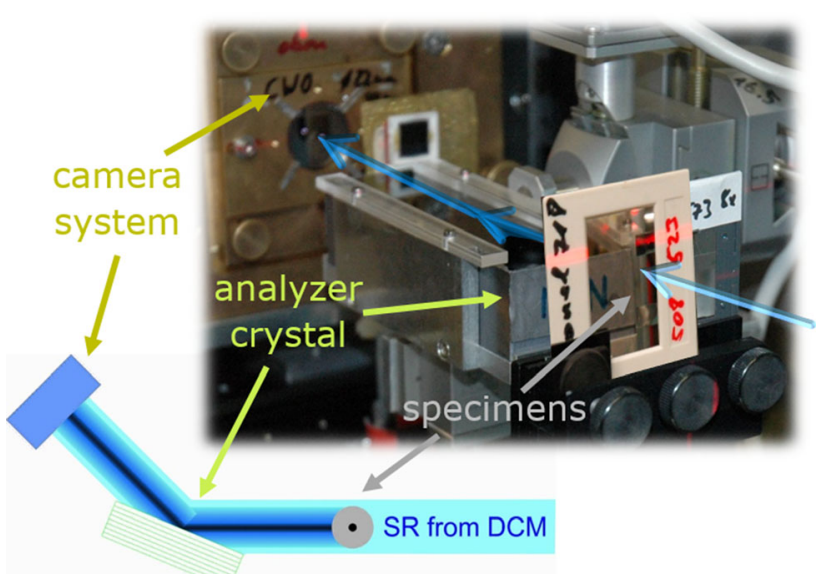

Fig. 2-Experimental set-up of the X-ray refraction station at the BAMline. The blue arrows indicate the beam path. The samples are mounted in a slide frame shown on the right. A sketch of the set-up is also indicated at the bottom of the photo (Color figure onine).

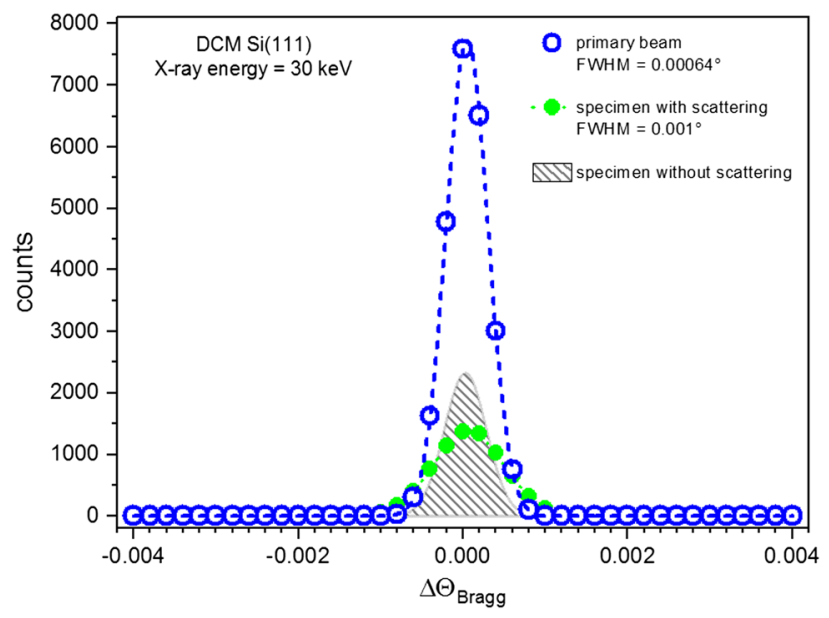

Fig. 3-Rocking curves measured at one (arbitrary) detector pixel. Open circles: without sample. Filled circles: with sample. The hatched area represents the calculated rocking curve assuming that the sample would not have any internal interfaces and thus would not display any refraction effect.

The background-corrected rocking curve images were analyzed using an in-house software code based on LabView ${ }^{\circledR}$. Figure 3 shows typical rocking curves extracted from one arbitrary detector pixel, with and without the sample in the beam. The intrinsic beam divergence was extracted from the rocking curve without the sample in place. The beam possesses a full width half maximum (FWHM) of about $0.00064 \mathrm{deg}$. The rocking curve with sample has an increased FWHM of about $0.001 \mathrm{deg}$, due to refraction at interfaces inside the sample. Without interfaces in the sample, the shaded curve in Figure 3 would have been measured. This means that the reduced peak height of the measured rocking curve (filled circles) is caused by both X-ray attenuation caused by the sample and X-ray refraction resulting from inner surfaces inside the sample. The reduced peak integral intensity (area beneath filled circles) is related to the attenuation properties of the sample. The analysis software calculates the values of the rocking curve integral, the peak height, the peak position, and the FWHM. The material's linear attenuation coefficient $\mu\left[\mathrm{mm}^{-1}\right]$ and the relative specific surface content of the samples are calculated from the quantities listed in Table II.

Using the image analysis software Fiji Image ${ }^{[29,30]}$ the attenuation properties $(\mu \cdot d)$ of a sample of thickness $d$, its refraction value $\left(C_{\mathrm{m}} \cdot d\right)$, and its relative specific surface $\left(C_{\mathrm{m}} / \mu\right)$ are evaluated for each pixel according to Eqs. [1] through [3]), respectively.

$$
\begin{gathered}
\mu \cdot d=-\ln \left(\frac{I}{I_{0}}\right) \\
C_{\mathrm{m}} \cdot d=1-\frac{I_{\mathrm{R}}}{I_{\mathrm{R} 0}} \cdot \frac{I_{0}}{I} \\
C_{\mathrm{m}} / \mu=\left(1-\frac{I_{\mathrm{R}}}{I_{\mathrm{R} 0}} \cdot \frac{I_{0}}{I}\right) /\left(-\ln \left(\frac{I}{I_{0}}\right)\right)
\end{gathered}
$$

A detailed description of the data conditioning and evaluation can be found. ${ }^{[31,32]}$

Note that the local relative specific internal surface of the sample is independent of its thickness $d$ and more importantly of its local variations. We notice that by analyzing the rocking curve, we separate the linear absorption coefficient by applying Eq. [1], and the refraction/scatter fraction by applying Eq. [3]. While 
Table II. Rocking Curve Parameter

\begin{tabular}{lll}
\hline Symbol & \multicolumn{1}{c}{ Quantity } & Physical Meaning \\
\hline$I_{\mathrm{R}}$ & $\begin{array}{c}\text { peak height with sample in the beam } \\
\text { (curve with filled circles) } \\
\text { peak height without sample in the beam } \\
\text { (curve with open circles) }\end{array}$ & $\begin{array}{c}\text { reduced maximum peak intensity caused by the attenuation } \\
\text { properties and internal interfaces of the sample } \\
\text { maximum peak intensity of the monochromatic } \\
\text { synchrotron beam }\end{array}$ \\
$\begin{array}{l}\text { peak integral with sample in the beam } \\
\text { (curve with filled circles) }\end{array}$ & $\begin{array}{c}\text { reduced peak intensity caused by the attenuation } \\
\text { properties of the sample } \\
\text { peak integral without sample in the beam } \\
\text { (curve with open circles) }\end{array}$ & $\begin{array}{c}\text { overall peak intensity of the monochromatic synchrotron beam } \\
I_{0}\end{array}$ \\
\hline
\end{tabular}

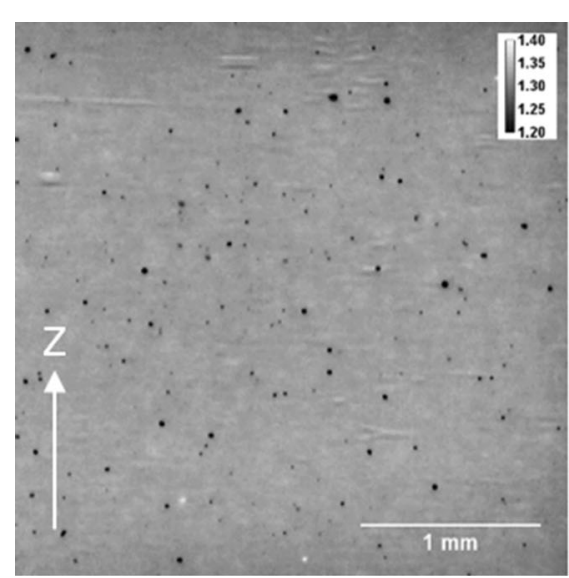

(a)

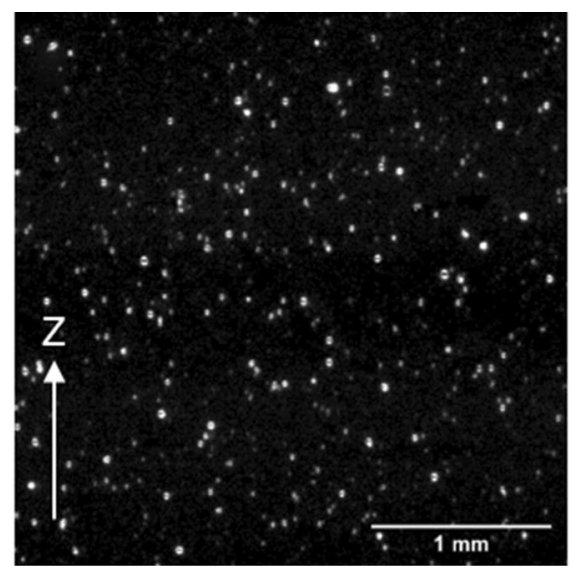

(b)

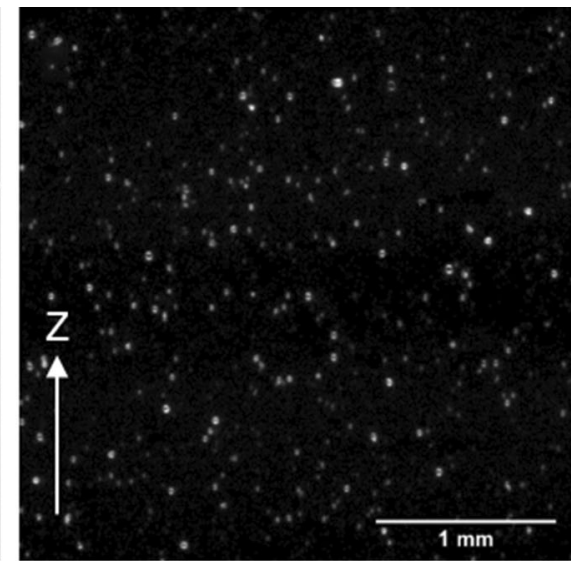

(c)

Fig. 4 -Example of data treatment steps for sample \#523 (non-HIPed with direct age). (a) Image of the attenuation property $(\mu \cdot d)$; $(b)$ refraction value $\left(C_{\mathrm{m}} \cdot d\right) ;(c)$ relative specific internal surface $\left(C_{\mathrm{m}} / \mu\right)$. The build direction $Z$ was oriented parallel to the scattering vector.

other works have dealt with the detailed character of the refracted signal (reflection, refraction, small angle scattering), see References 33 and 34, the correlation between X-ray refraction signal and specific surface has been previously quantitatively established. ${ }^{[13,14]}$ This is based on the hypothesis that the main scattering mechanism is refraction.

Figure 4 shows an example of the above-mentioned quantities, calculated for sample \#523 using Eqs. [1] through [3], as two-dimensional gray value images.

The local relative specific surface combined with the local attenuation properties (also obtained from SXRR) was used to identify the discontinuities observed. Irregular discontinuities aligned perpendicular to the build direction are indicative of lack of melt in-between successive layers. This type of discontinuity can result from non-optimized build parameters such as layer thickness or hatch spacing, or irregularities in the laser beam. If a large refraction signal is displayed, it indicates the region possesses a large relative specific surface within its volume. This would result from a complex shape or some type of internal structure, expected to be inclusions from entrapped splatter or unmelted powder in an AM sample. Crescent-shaped structures in the relative specific surface image correspond to circular-shaped voids with no internal structure, such as the case of empty voids (which can be caused by trapped gas).

\section{RESULTS AND DISCUSSION}

A comparison was made of the non-HIPed direct age vs the HIP two step age as various studies have shown that similar mechanical properties can be obtained with shorter heat treatments of Inconel 718. ${ }^{[23,24]}$ The particle size analysis of the starting powders showed a Gaussian distribution from 10 to $70 \mu \mathrm{m}$. The median size was about $27 \mu \mathrm{m}$ with a mean of $28 \mu \mathrm{m}$, and an aspect ratio of 0.98 . The density obtained from the Pycnometry measurements was $8.14 \mathrm{~g} / \mathrm{cm}^{3}$. This is slightly less than the reported $8.19 \mathrm{~g} / \mathrm{cm}^{3}$ reported for wrought Inconel 718, and corresponds to a void volume of 0.5 pet.

The binarized optical microscopy images of representative un-etched samples are shown in Figure 5. Table III summarizes the void sizes and area fractions measured. In the as-built and direct aged non-HIPed samples, almost round voids are observed that are similar in size and area fraction. After the heat treatment with HIP, the voids are reduced in both size and area fraction but are slightly elongated.

Optical microscopy images of corresponding representative etched samples are shown in Figure 6. Evidence of columnar grains is apparent in samples 554 and 523, neither of which were HIPed. Strong textures, evident in the almost uniform response to etching, have been correlated with this columnar grain structure in the 


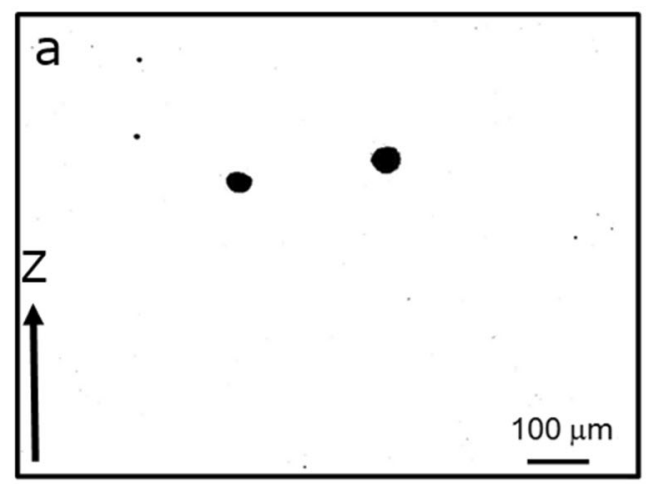

554 Build direction $(Z)$
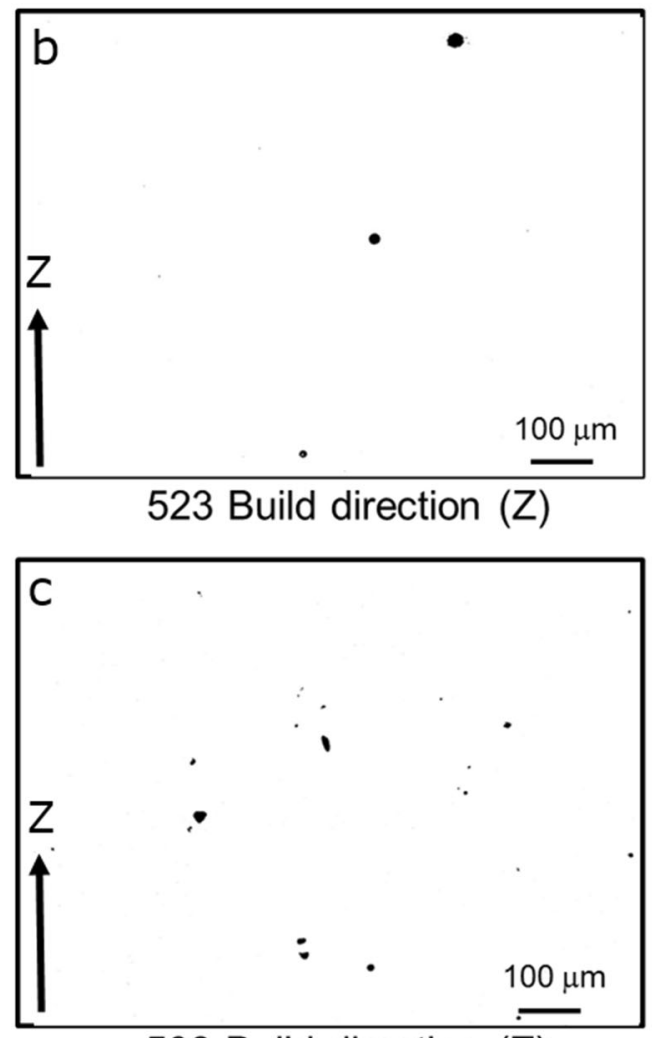

508 Build direction (Z)

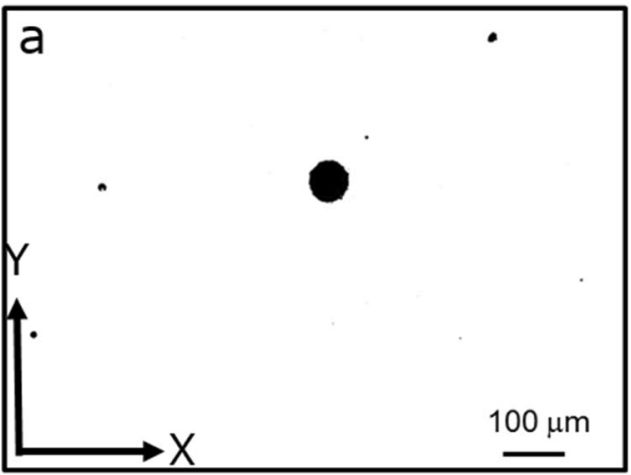

554 Build plane $(X-Y)$
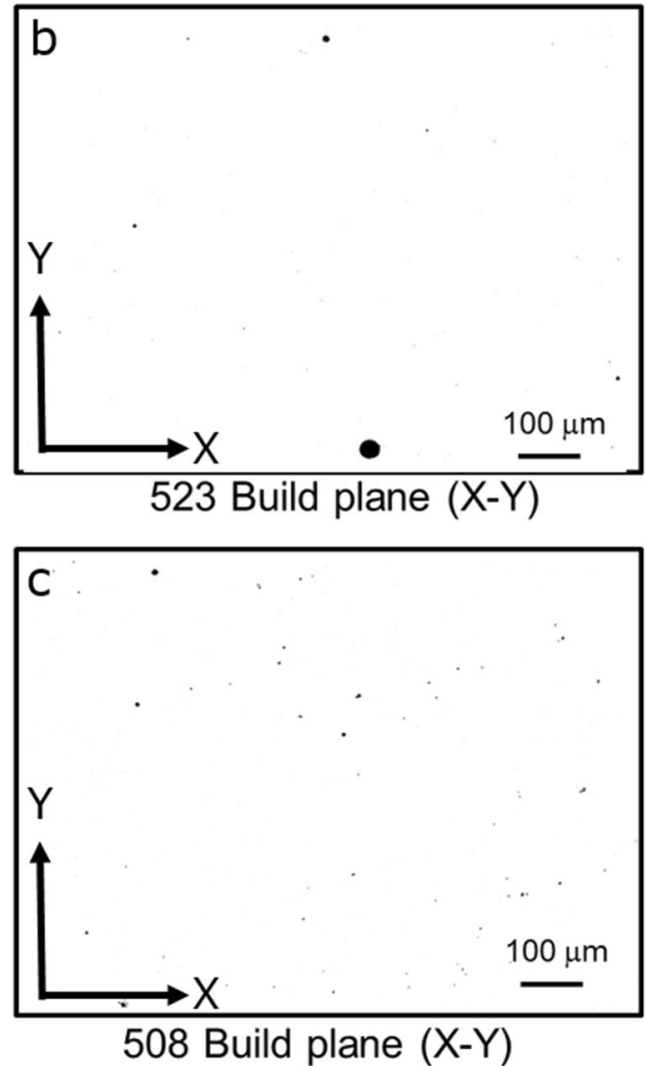

Fig. 5-Representative optical microscopy images of the un-etched samples. (a) Non-HIPed as-built (\#554), (b) non-HIPed and direct aged (\#523), and (c) HIP and heat treated per SAE/AMS 5663 (\#508).

Table III. Summary of Void Size Based on Optical Microscopy

\begin{tabular}{lccc}
\hline Sample ID & Area Fraction $($ Pct $)$ & Maximum Void Size $(\mu \mathrm{m})$ & Average Void Size $(\mu \mathrm{m})$ \\
\hline $554(X Y)$ & 0.29 & 64.5 & $12.3 \pm 13.9$ \\
$554(Z)$ & 0.17 & 50.0 & $11.7 \pm 12.5$ \\
$523(X Y)$ & 0.30 & 77.7 & $14.0 \pm 16.1$ \\
$523(Z)$ & 0.21 & 67.8 & $14.6 \pm 14.7$ \\
$508(X Y)$ & 0.15 & 39.0 & $5.3 \pm 4.5$ \\
$508(Z)$ & 0.15 & 43.2 & $5.4 \pm 5.4$ \\
\hline
\end{tabular}

Z build direction. ${ }^{[35-37]}$ Due to the retention of the scan pattern, grain sizes cannot be determined from the etched optical images.
After a full heat treatment including HIP, equiaxed grains are observed in both the build direction $(Z)$ and build plane (XY). The grains are on average $20 \mu \mathrm{m}$, with 


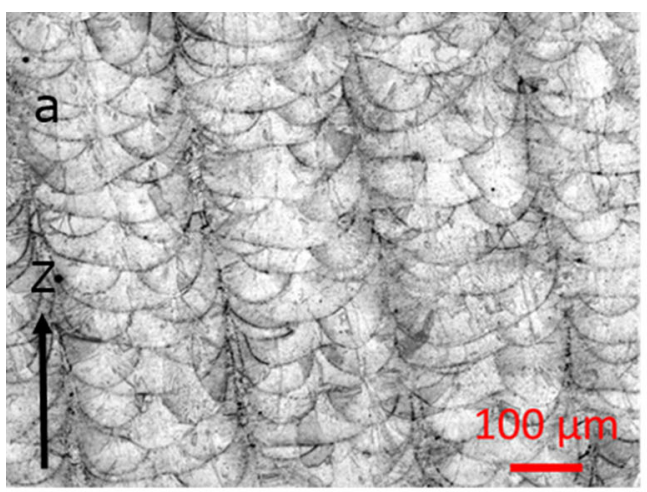

554 Build direction (Z)

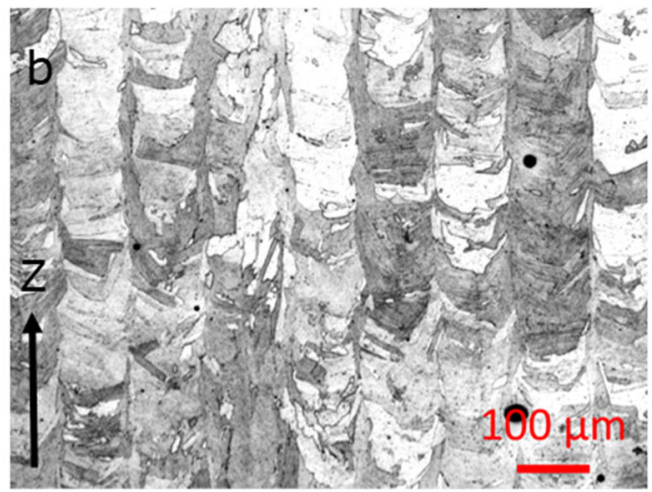

523 Build direction (Z)

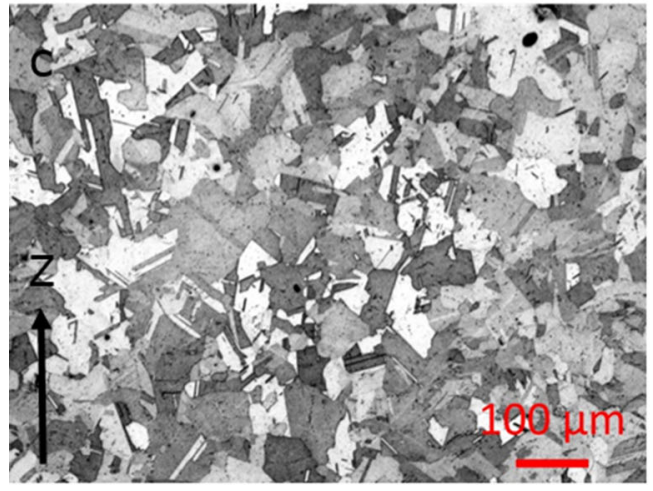

508 Build direction (Z)

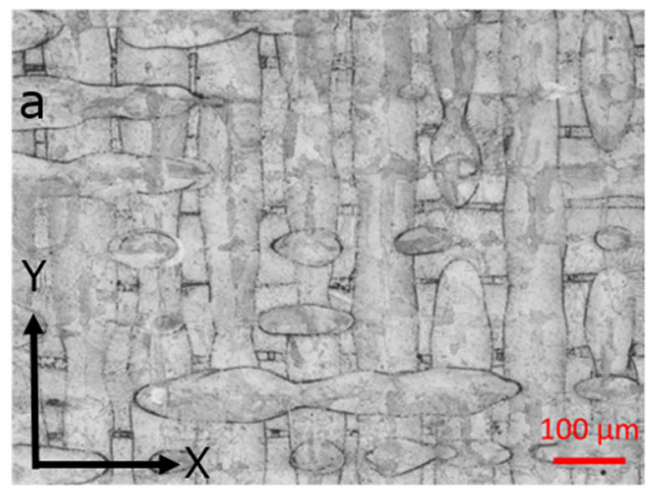

554 Build plane (X-Y)

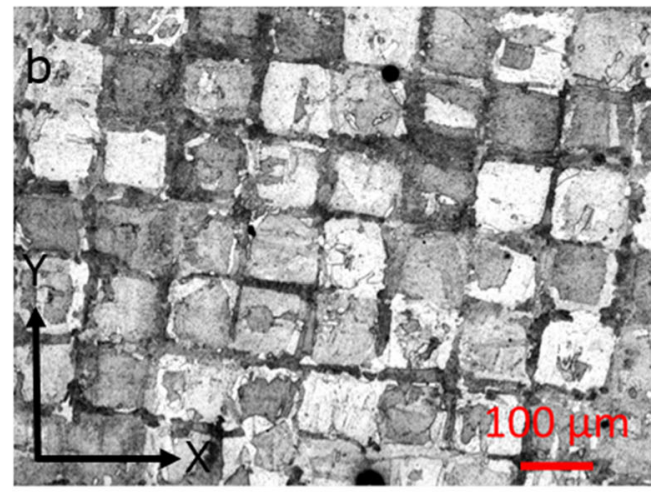

523 Build plane (X-Y)

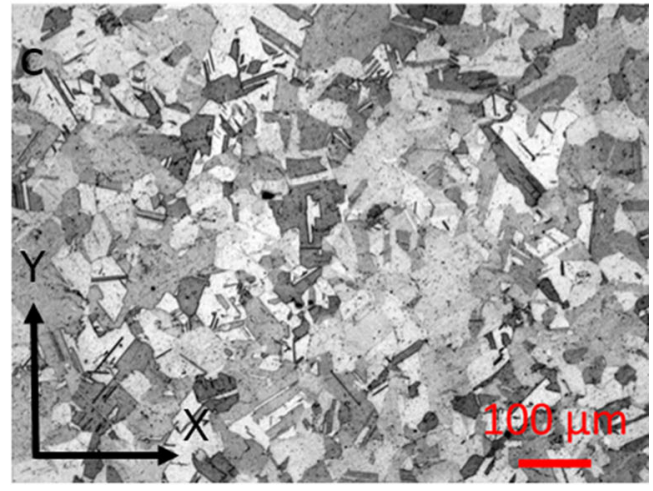

508 Build plane $(X-Y)$

Fig. 6 - Optical microscopy images of the etched samples. (a) Non-HIPed as-built (554), (b) non-HIPed and direct age (523), and (c) HIP and heat treated per SAE/AMS 5663.

several large grains on the order of $430 \mu \mathrm{m}$ in both orientations. Equiaxed grains imply that the texture has been randomized, as evidenced by the random variation in response to the etching.

The local values of $\mu \cdot d$ are visualized as $2 \mathrm{D}$ gray scale images in Figure 7 (left) for sample non-HIPed and direct age sample \#523 and in Figure 8 for the HIPed and heat-treated sample \#508 oriented with its build direction $Z$ parallel (top) and perpendicular (bottom) to the scattering plane, respectively. A homogenous $\mu \cdot d$ value is observed across both samples (the edges show exceptions due to the polishing artifacts). The
non-HIPed and heat-treated sample \#523 reveals dark circular spots of apparent low density. The average size of these dark spots is approximately $40 \mu \mathrm{m}$. Such spots are visible at the same location in both orientations of the sample as shown by rectangles no. 1 and 2 in Figure 7 (left, top and bottom), indicating identical positions within the sample. The dark spots correspond to the bright spots in the of $C_{\mathrm{m}} / \mu$ images as shown in Figure 7, middle, top and bottom images. The enlargement of rectangle no. 1 in Figure 7 right, shows crescents in both orientations at the upper and lower edges of the voids, while their center is dark. This 

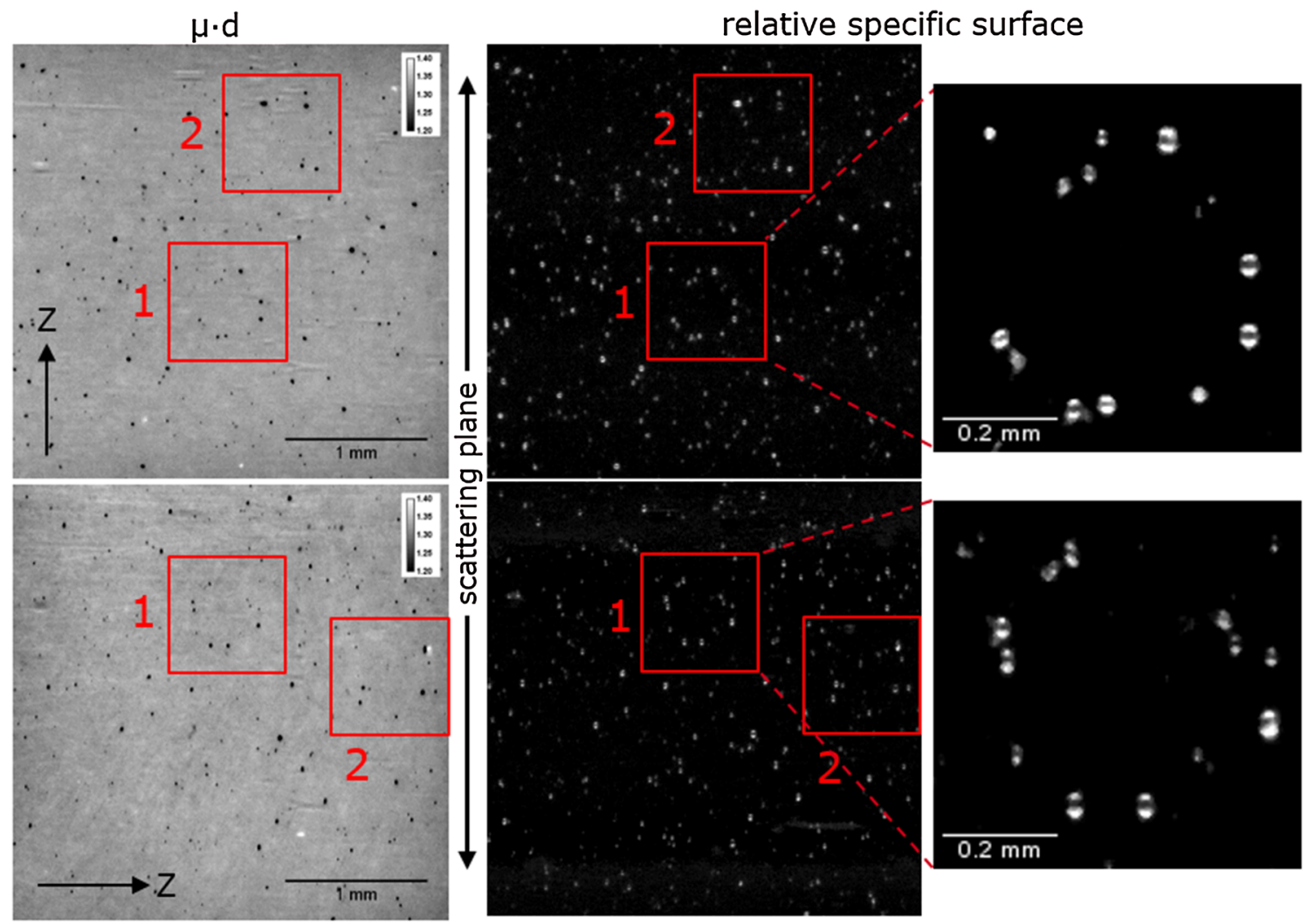

Fig. 7- Comparison of two orientations of the build direction $Z$ for the non-HIPed and direct aged sample \#523 with respect to the scattering plane of the detector system (Top - parallel, bottom - perpendicular). Left: $\mu \cdot d$ (attenuation), middle: specific surface (refraction), right: shows the enlargement of the rectangle no. 1. Numerical values are given in Table IV.

indicates, that the dark spots in the $\mu \cdot d$ images are empty voids or cavities (i.e., not filled with unmelted powder, or representing high-density grains). There is pronounced orientation dependence of the various features observed. While the $\mu \cdot d$ image is independent of the sample orientation, the relative specific surface image has a slight dependency. The crescents seem to be slightly elongated in the parallel orientation than in the perpendicular orientation as shown by comparison with Figure 7 (right top and bottom). This is also suggested by the values reported in Table IV. This means that the discontinuities or cavities are slightly elongated, and oriented along the build plane $X-Y$. This slight elongation in the build plane could result from non-optimized hatch spacing used in the build or variations in laser power.

In contrast, these small dark circular spots are not observed in the HIPed and heat-treated sample \#508 shown in the attenuation images in Figure 8. Rather, bright spots of larger size, with apparent high density, are observed. Such bright spots have irregular boundaries and are not stationary with respect to the sample as noted by comparison with the rectangle no. 1 shown in (left, top and bottom images). Thus, they do not correspond to dense regions, but rather to grains that are oriented to fulfill the Bragg condition for the monochromatic radiation used. This phenomenon removes intensity from the transmitted beam so that these regions appear as absorbing or bright. In fact these grains do not fulfill the Bragg condition if the sample is rotated $90 \mathrm{deg}$. They can, however, be used to estimate the size of crystallites or coherent domains. The size of these bright spots is approximately $200 \mu \mathrm{m}$. This corresponds to the largest grain sizes observed in the equiaxed sample that was HIPed and heat treated (see Figure 6(c) left). We also notice that, since the sample thickness of the SXRR measurement is $160 \mu \mathrm{m}$, the spots in Figure 8 correspond to single crystallites. Such bright spots are not observed in the attenuation maps of sample \#523 simply because the grain orientation is not favorable.

The comparison between the $\mu \cdot \mathrm{d}$ and $C_{\mathrm{m}} / \mu$ image for the HIPed and heat-treated sample \#508 does not show the single voids or cavities that were visible in Figure 7 for the non-HIPed and direct aged sample \#523. Rather some bright features are visible in the $\mu \cdot \mathrm{d}$ image which possess a certain amount of relative specific surface that relates to grain or sub-grain boundaries and/or to forests of dislocations (similar structures have been observed in Al alloys). ${ }^{[32,38]}$ The very weak and blurred refraction intensity in the relative specific surface image, shown by the magnified image in Figure 8 right, is thus probably related to grain boundaries oriented perpendicular to the scattering vector, or alternatively to pores that have not closed after HIPing.

In the optical microscopy images, the void area and size are similar in the non-HIPed samples for both the as-built and the direct age. Due to their round shape and 

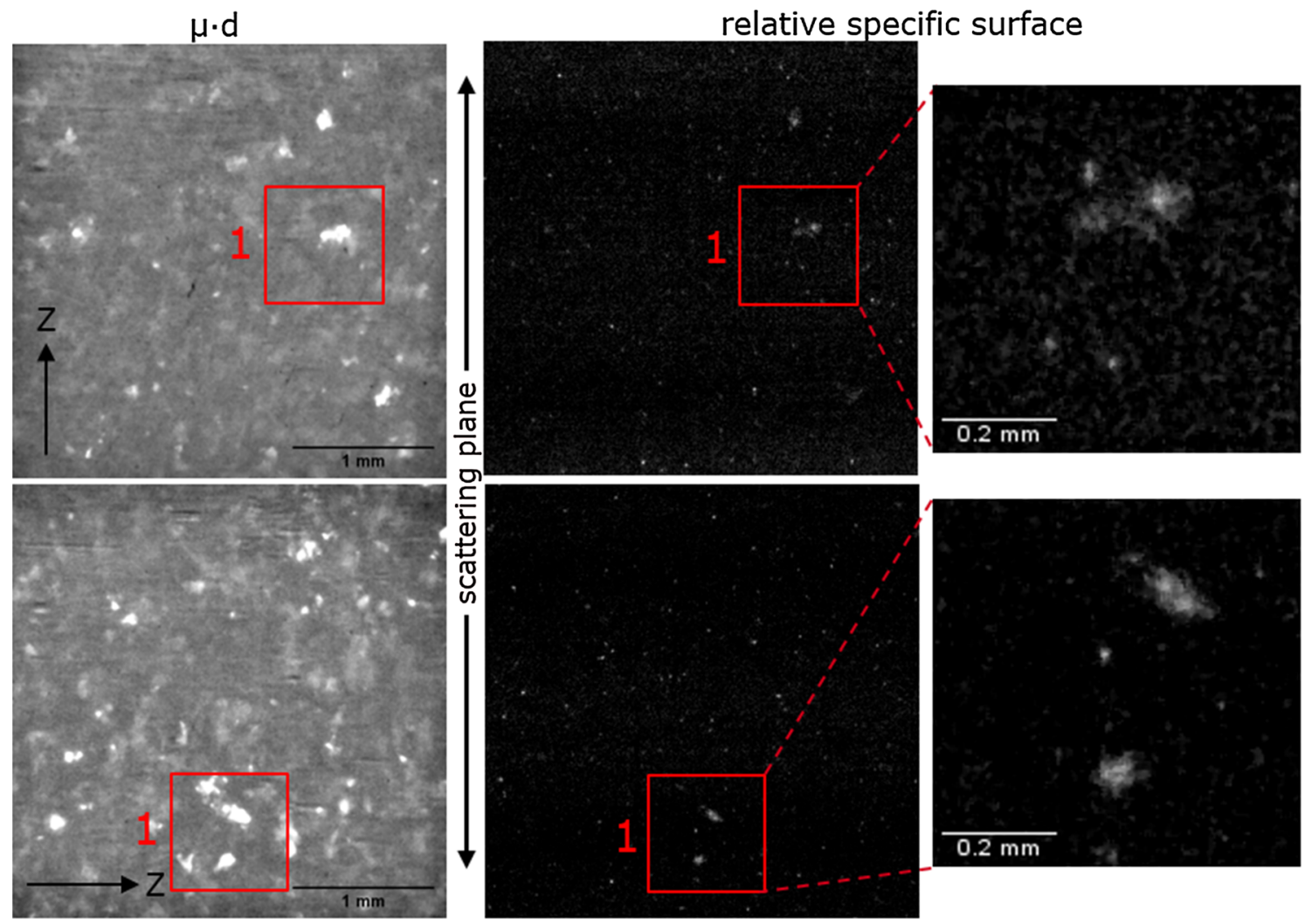

Fig. 8 - Comparison of two orientations of the HIPed and heat-treated sample \#508 with respect to the scattering vector (Top-parallel, bottom - perpendicular). Left: $\mu \cdot d$ (attenuation), middle: specific surface (refraction), right: enlargement of the rectangle no. 1, which marks the same area of the sample.

smooth interior surface, these may be retained from initial porosity in the argon gas atomized powders used in the build. ${ }^{[1,10,39-41]}$ Analysis of the powders indicates an 'initial' void volume of around 0.5 pet. This is slightly higher than the maximum measured porosity by optical microscopy $(0.3 \mathrm{pct})$. Considering the maximum voids measured with optical microscopy and SXRR, a $14 \mu \mathrm{m}$ diameter void could easily reside within the $70 \mu \mathrm{m}$ maximum size of the starting powder. This would indicate voids present in the gas atomized powder could be retained in the AM build and do not necessarily result from the AM process. The slight increase in size of the void in the non-HIPed sample after heat treatment would result from the expansion of trapped gas. Obviously, a detailed analysis of the origin of the pores would need in situ monitoring (e.g., by means of active thermography ${ }^{[42,43]}$ ), but this is outside the scope of this work.

Table IV summarizes the mean values (integrated across the field-of-view) of $\mu \cdot d, \mu, d$ and $C_{\mathrm{m}} / \mu$ of the two samples in the two orientations. Differences in $\mu \cdot d$ are not solely due to the variation of $d$ between the two samples. Using their average measured thickness, a mean value of $\mu$ for each sample could be calculated as shown in Table IV. As expected, the measured value of $\mu \cdot d$ or $\mu$ is invariant against sample orientation. Remarkably, the attenuation coefficient is different in the two materials; this can be due not only to the different amount of porosity but also to the presence of strongly diffracting grains in the equiaxed sample \#508.

The values of the relative specific surface are small for both samples in both orientations. In spite of the fact that such values lie just above the detection limit of the experimental set-up, it can be stated that the non-HIPed direct aged sample \#523 has a two times larger value of the relative specific surface compared to the HIPed and aged sample \#508. In both samples, the perpendicular orientation shows smaller values of $C_{\mathrm{m}} / \mu$, thereby indicating some preferential discontinuity alignment with the powder layer plane. This could result from non-optimized hatch spacing or variations in laser power during the build.

The results of the mechanical tests are summarized in Table V. The non-HIPed as-built sample has the lowest strength, as expected for the heat treatable Inconel 718 alloy with a corresponding larger elongation at fracture. Although the area fraction of voids and their size and morphology are similar between the non-HIPed as-built \#554 and the heat-treated \#523, the elongation at fracture is notably affected. This is expected as increases in strength are usually accompanied by a reduction in ductility. This implies that porosity is not the most important factor determining the mechanical properties in uniaxial static tests.

The heat-treated and HIPed sample \#508 has both the highest strength and elongation at fracture with the lowest void area and size. Since the strength of the 
Table IV. Linear Attenuation Coefficient and Relative Internal Specific Surface Calculated from X-ray Transmission and Refraction Measurements

\begin{tabular}{lccrr}
\hline Sample & Orientation of Build Direction to the Scattering Vector & $\mu \cdot d$ & $d(\mathrm{~mm})$ & $\mu(\mathrm{mm})$ \\
\hline$\# 508$ & parallel & 1.629 & 0.17 & 9.850 \\
$\# 508$ & perpendicular & 1.632 & 0.00183 \\
$\# 523$ & parallel & 1.320 & 0.15 & 9.602 \\
$\# 523$ & perpendicular & 1.323 & 8.803 & 0.00074 \\
\hline
\end{tabular}

Table V. Mechanical Properties of Samples

\begin{tabular}{lcrr}
\hline Sample ID & Ultimate Tensile Stress (MPa) & Yield Stress (MPa) & Elongation at Fracture (Pct) \\
\hline 554 & $995 \pm 13$ & $698 \pm 15$ & $33.2 \pm 1.10$ \\
523 & $1362 \pm 12$ & $1233 \pm 12$ & $19.1 \pm 0.74$ \\
508 & $1406 \pm 7$ & $1170 \pm 6$ & $24.9 \pm 0.39$ \\
\hline
\end{tabular}

HIPed and aged sample is similar to the non-HIPed and direct aged sample, the size, area fraction and morphology do not greatly affect the quasi-static strength. However, it should be noted that since the morphology of the voids changed as a result of the HIP process, the effect of the elongated voids maybe more pronounced in dynamic fatigue tests. The slightly lower ductility measured in quasi-static tension tests may also correspond to decreased fatigue life. Interestingly, a primary difference between the non-HIPed and HIPed processes is the higher temperature of the HIP process of $1163^{\circ} \mathrm{C}$. It is this higher temperature that is attributed to forming the refined equiaxed grain morphology observed in the HIPed and aged sample \#508. In fact, in spite of the high temperature, no grain growth occurs during HIPing. This is attributed to grain boundary pinning by carbides or precipitates which limit grain boundary mobility.

\section{SUMMARY}

The use of $2.5 \mathrm{D}$ synchrotron radiation refraction radiography (SXRR), yielding both attenuation and refraction information, allows a great deal of insight into the nature of the discontinuities observed in the microstructure of AM SLM Inconel 718. Samples were investigated in the non-HIPed as-built, non-HIPed direct age and HIPed with two step aging treatments. Although the differences in the quasi-static tension tests were attributed to the aging heat treatments to promote precipitate formation and subsequent grain refinement in age hardenable alloys rather than a difference in the nature of the discontinuities within the samples, the nature of the discontinuities is expected to affect the dynamic fatigue life.

Optical micrographs show a slight increase in void size and area fraction in the non-HIPed samples after heat treatment which could be due to expansion of trapped gases. The average $14 \mu \mathrm{m}$ void size with a maximum of $70 \mu \mathrm{m}$ in the non-HIPed direct aged sample decreased to a $5 \mu \mathrm{m}$ average size with a maximum average of $40 \mu \mathrm{m}$ in the HIPed samples after the two step aging heat treatment. X-ray attenuation images of the non-HIPed and direct age sample show 40 $\mu \mathrm{m}$ dark spots that correspond to very low-density objects with smooth circular boundaries that can be identified as voids. In the relative specific surface image these discontinuities correspond to juxtaposed crescents surrounding a dark center indicating the objects are empty voids, which correlate with the 2D optical microscopy images.

From the SXRR, the specific surface value of the non-HIPed sample was about 2 times higher than the HIPed sample. This effect is ascribed to the differences in the porosity ranging from 0.20 to 0.30 pct for the non-HIPed samples, with respect to the $0.15 \mathrm{pct}$ in the HIPed sample. The SXRR also detected a change in morphology from a circular shape to a slightly elongated shape in the HIPed sample. The change in grain morphology, size and area fraction did not appreciably change the quasi-static tensile strength although is expected to be more pronounced in dynamic fatigue tests.

Noticeable differences in the microstructure were observed in the optical images of the non-HIPed and HIPed samples. After the HIP and aging heat treatment, the microstructure consisted of refined, equiaxed grains with an average grain size of $20 \mu \mathrm{m}$ and maximum of $430 \mu \mathrm{m}$. Although similar strengths were noted in the non-HIPed direct age and HIPed two step age, the ductilities differ. The higher ductility in the HIPed sample is attributed to the refined equiaxed grains. A noted difference between the heat treatments was the HIP temperature of $1163{ }^{\circ} \mathrm{C}$ as compared to the homogenization/solutionizing temperature of $1066{ }^{\circ} \mathrm{C}$. Whether this higher temperature triggers the grain refinement $v s$ the pressure of the HIP process is unknown as mechanisms driving grain refinement in AM processing remain under investigation.

X-ray attenuation images of the HIP process and two step age sample shows bright spots about $200 \mu \mathrm{m}$ in size 
with irregular boundaries. The bright spots in the attenuation images also display a large relative specific surface, related to grain or sub-grain boundaries and/or forests of dislocations and are thus correlated with specifically oriented grains. The non-homogenous distribution of these bright spots correlates with the random orientation of the equiaxed grains. Similar characteristics are not observed in the non-HIPed direct aged sample which retains columnar grains.

SXRR also identified discontinuities with preferential alignment along the powder layers. While this characteristic was observed in both the SXRR analysis of the non-HIPed and HIPed sample, it was not observed in the optical microscopy images. These types of discontinuities could result from non-optimized hatch spacing or variations in laser power during the build. Understanding and mitigating the cause of this discontinuity, which persisted after the HIP process, is critical to the structural integrity of AM components.

\section{CONCLUSIONS}

For optimization of AM processing parameters, an understanding of the underlying mechanisms of discontinuity formation is required. The use of non-destructive synchrotron $\mathrm{X}$-ray refraction and transmission radiography has been shown to be an extremely valuable tool in identifying the nature of discontinuities in a build, especially when anchored with optical microscopy. As this study showed, quasi-static mechanical properties are not sufficient to quantify the quality of a printed AM part. Although optimized parameters were used in the build of the specimens in this study, lack of melt in indications were observed which did not heal during the HIP process. Once identified, further parameter development can alleviate this discontinuity. Other discontinuities such as voids could be easily distinguished due to their lack of internal surface features. Additional information is also obtained regarding grain morphology, size and texture. Since the field-of-view investigated by $\mathrm{X}$-ray refraction techniques is much larger than that obtainable by optical microscopy, this technique yields better statistics and can be readily correlated with the mechanisms of discontinuity formation. Expanding this study to the rationalization of dynamic mechanical properties in fatigue tests due to differences in discontinuity formation is left for future work.

\section{ACKNOWLEDGMENTS}

Open Access funding provided by Projekt DEAL. We thank HZB colleagues for their support as well as Ralf Britzke and Thomas Wolk (BAM) for their assistance during beam time at BAMline. One author (JAS) acknowledges the opportunity as a guest scientist at the Federal Institute for Materials Research and Testing (BAM) to participate in this collaborative research.

\section{OPEN ACCESS}

This article is licensed under a Creative Commons Attribution 4.0 International License, which permits use, sharing, adaptation, distribution and reproduction in any medium or format, as long as you give appropriate credit to the original author(s) and the source, provide a link to the Creative Commons licence, and indicate if changes were made. The images or other third party material in this article are included in the article's Creative Commons licence, unless indicated otherwise in a credit line to the material. If material is not included in the article's Creative Commons licence and your intended use is not permitted by statutory regulation or exceeds the permitted use, you will need to obtain permission directly from the copyright holder. To view a copy of this licence, visit http://creat ivecommons.org/licenses/by/4.0/.

\section{REFERENCES}

1. S. Tammas-Williams, P.J. Withers, and P.B. Prangnell: Scripta Mater., 2016, vol. 122, pp. 72-76.

2. C. Schaak, W. Tillmann, M. Schaper, and M.E. Sydinoez: Forum Rapid Technol., 2016, vol. 13, p. 4403.

3. B. Zhang, Y. Li, and Q. Bai: J. Chin. J. Mech. Eng., 2017, vol. 30, pp. $515-27$

4. S. Siddique, M. Imran, M. Rauer, M. Kaloudis, E. Wycisk, C. Emmelmann, and F. Walther: Mater. Des., 2015, vol. 83, pp. 661-69.

5. A. Yadollahi and N. Shamsaei: Int. J. Fatigue, 2017, vol. 98, pp. 14-31.

6. S. Beretta and S. Romano: Int. J. Fatigue, 2017, vol. 94, pp. $178-91$.

7. R.C. Cooper, G. Bruno, Y. Onel, A. Lange, T.R. Watkins, and A. Shyam: J. Mater. Sci., 2016, vol. 51, pp. 9749-60.

8. T. Thiede, T. Mishurova, S. Evsevleev, I. Serrano-Munoz, C. Gollwitzer, and G. Bruno: Quantum Beam Sci., 2019, vol. 3, pp. $1-12$.

9. B.R. Müller, R.C. Cooper, A. Lange, A. Kupsch, M. Wheeler, M.P. Hentschel, A. Staude, A. Pandey, A. Shyam, and G. Bruno: Acta Mater., 2018, vol. 144, pp. 627-41.

10. G. Kasperovich, J. Haubrich, J. Gussone, and G. Requena: Mater. Des., 2016, vol. 105, pp. 160-70.

11. A. Kupsch, B.R. Müller, A. Lange, and G. Bruno: J. Eur. Ceram. Soc., 2017, vol. 37, pp. 1879-89.

12. M.P. Hentschel, R. Hosemann, A. Lange, B. Uther, and R. Bruckner: Acta Crystallogr. A, 1987, vol. 43, pp. 506-13.

13. F.E. Fensch-Kleemann, K.-W. Harbich, and M.P. Hentschel: Ceram. Forum Int., 2002, vol. 79, pp. E35-38.

14. K.W. Harbich, M.P. Hentschel, and J. Schors: NDT \& E Int., 2001, vol. 34, pp. 297-302.

15. B.R. Müller and M.P. Hentschel: X-Ray Spectrom., 2004, vol. 33, pp. $402-06$.

16. R. Laquai, T. Schaupp, B.R. Müller, A. Griesche, G. Bruno, and T. Kannengießer: in NDT-CE 2015 - International Symposium Non-destructive Testing in Civil Engineering (Proceedings), Technische Universität Berlin / Bundesanstalt für Materialforschung und -prüfung: Berlin, Germany, 2015, pp. 1217-24.

17. R. Laquai, B.R. Müller, G. Kasperovich, J. Haubrich, G. Requena, and G. Bruno: Mater. Res. Lett., 2018, vol. 6, pp. 130-35.

18. B.R. Müller and M.P. Hentschel: in Handbook of Technical Diagnostics-Fundamentals and Application to Structures and Systems, H. Czichos, ed., Springer, 2013, pp. 287-300.

19. S. Günzel, S. Hickmann, C. Wittemeyer, and V. Trappe: Adv. Eng. Mater., 2012, vol. 14, pp. 867-72.

20. R.C. Cooper, G. Bruno, M.R. Wheeler, A. Pandey, T.R. Watkins, and A. Shyam: Acta Mater., 2017, vol. 135, pp. 361-71. 
21. A. Kupsch, A. Lange, M.P. Hentschel, Y. Onel, T. Wolk, A. Staude, K. Ehrig, B.R. Müller, and G. Bruno: J. Ceram. Sci. Technol., 2013, vol. 4, pp. 169-76.

22. SAE/AMS 5664, 2009.

23. J.A. Schneider, B. Lund, and M. Fullen: $A M$ J., 2018, vol. 21, pp. $248-54$

24. I. Kelbassa, E.W. Kreutz, P. Albus, and L. Zhu: in Proc. 24th Intl. Congr. Appl. Lasers \& Electro-optics (ICALEO), Laser Institute of America, Orlando, pp. 660-65.

25. B.R. Müller, A. Lange, M. Harwardt, and M.P. Hentschel: $A d v$. Eng. Mater., 2009, vol. 11, pp. 435-40.

26. W. Görner, M.P. Hentschel, B.R. Müller, H. Riesemeier, M. Krumrey, G. Ulm, W. Diete, U. Klein, and R. Frahm: Nucl. Instrum. Methods Phys. Res. Sect. A, 2001, vol. 467, pp. 703-06.

27. A. Rack, S. Zabler, B.R. Müller, H. Riesemeier, G. Weidemann, A. Lange, J. Goebbels, M. Hentschel, and W. Görner: Nucl. Instrum. Methods A, 2008, vol. 586, pp. 327-44.

28. A. Lange, M.P. Hentschel, A. Kupsch, and B.R. Müller: Int. J. Mater. Res., 2012, vol. 103, pp. 174-78.

29. Wayne Rasband, 1997.

30. C.A. Schneider, W.S. Rasband, and K.W. Eliceiri: Nat. Methods, 2012, vol. 9, p. 671.

31. J. Nellesen, R. Laquai, B.R. Müller, A. Kupsch, M.P. Hentschel, N.B. Anar, E. Soppa, W. Tillmann, and G. Bruno: J. Mater. Sci., 2018, vol. 53, pp. 6021-61.

32. Sandra Cabeza, Bernd Randolf Müller, Rocío Pereyra, Ricardo Fernandez, G. Gonzalez-Doncel and Giovanni Bruno, Journal of Applied Crystallography 2018, vol. 51, p. https://doi.org/10.1107/ S1600576718001449.
33. F. Zhang, G.G. Long, L.E. Levine, J. Ilavsky, and P.R. Jemian: J. Appl. Crystallogr. 2008, vol. 41.

34. O. Oltulu, Z. Zhong, M. Hasnah, M. Wernick, and D. Chapman: J. Phys. D, 2003, vol. 36, p. 2152.

35. L.N. Carter, C. Martin, P.J. Withers, and M.M. Attallah: J. Alloys Compd., 2014, vol. 615, pp. 338-47.

36. N. Nadammal, S. Cabeza, T. Mishurova, T. Thiede, A. Kromm, C. Seyfert, L. Farahbod, C. Haberland, J.A. Schneider, P. Portella, and G. Bruno: Mater. Des., 2017, vol. 134, pp. 139-50.

37. L. Yanjin, W. Songquan, Y. Gan, T. Huang, C. Yang, J. Lin, and J. Lin: Opt. Laser Technol., 2015, vol. 75, pp. 197-206.

38. R. Fernández, G. Bruno, and G. González-Doncel, 2018, vol. 123, p. 145108 .

39. W.J. Sames, F.A. List, S. Pannala, R.R. Dehoff, and S.S. Babu: Int. Mater. Rev., 2016, vol. 61, pp. 315-60.

40. W.J. Sames, F. Medina, W.H. Peter, S.S. Babu, and R.R. Dehoff: in Superalloy 718 \& Derivatives, Pittsburgh, PA, 2014

41. H. Qi, M. Azer, and A. Ritter: Metall. Mater. Trans. A, 2009, vol. 40A, pp. 2410-22.

42. N. Scheuschner, S. Altenburg, A. Straße, A. Gumenyuk, and C. Maierhofer: in II International Conference on Simulation for Additive Manufacturing-Sim-AM 2019, Pavia, Italy, 2019, pp. 246-55.

43. U. Hassler, D. Gruber, O. Hentschel, F. Sukowski, T. Grulich, and L. Seifert: Phys. Procedia, 2016, vol. 83, pp. 1244-52.

Publisher's Note Springer Nature remains neutral with regard to jurisdictional claims in published maps and institutional affiliations. 